\title{
Dirac plus Nambu monopoles in the Standard Model
}

\author{
George Lazarides@, ${ }^{1}$ Qaisar Shafi, ${ }^{2}$ and Tanmay Vachaspati ${ }^{3}$ \\ ${ }^{1}$ School of Electrical and Computer Engineering, Faculty of Engineering, \\ Aristotle University of Thessaloniki, Thessaloniki 54124, Greece \\ ${ }^{2}$ Bartol Research Institute, Department of Physics and Astronomy, University of Delaware, \\ Newark, Delaware 19716, USA \\ ${ }^{3}$ Physics Department, Arizona State University, Tempe, Arizona 85287, USA
}

(Received 16 June 2021; accepted 6 August 2021; published 20 August 2021)

\begin{abstract}
We show how in the standard electroweak model three $S U(2)_{L}$ Nambu monopoles, each carrying electromagnetic (EM) and $Z$ magnetic fluxes, can merge (through $Z$ strings) with a single $U(1)_{Y}$ Dirac monopole to yield a composite monopole that only carries EM magnetic flux. Compatibility with the Dirac quantization condition requires this composite monopole to carry six quanta $(12 \pi / e)$ of magnetic charge, independent of the electroweak mixing angle $\theta_{w}$. The Dirac monopole is not regular at the origin and the energy of the composite monopole is therefore divergent. We discuss how this problem is cured by embedding $U(1)_{Y}$ in a grand unified group such as $S U(5)$. A second composite configuration with only one Nambu monopole and a colored $U(1)_{Y}$ Dirac monopole that has minimal EM charge of $4 \pi / e$ is also described. Finally, there exists a configuration with an EM charge of $8 \pi / e$ as well as screened color magnetic charge.
\end{abstract}

DOI: 10.1103/PhysRevD.104.035020

\section{INTRODUCTION}

It is widely recognized that the Standard Model (SM) based on the gauge group $S U(3)_{c} \times S U(2)_{L} \times$ $U(1)_{Y} / Z_{3} \times Z_{2}$ does not contain topologically stable finite-energy monopoles. This stems from the fact that the vacuum manifold in the SM is a three-sphere and does not have a nontrivial second homotopy group that is required for the existence of topological finite-energy monopoles. Note that this result is unaffected if additional scalar and/or matter fields are introduced in the SM. Nevertheless, as we show, interesting composite topological structures are present in the SM and its extensions such as grand unified theories (GUTs).

It was recognized sometime ago [1-3] that a 't HooftPolyakov-type [4,5] monopole configuration can be realized within the $S U(2)_{L}$ sector of the SM. This is achieved by utilizing a Higgs doublet field configuration which effectively behaves like a scalar $S U(2)_{L}$ triplet with hypercharge $Y=0$. This yields a monopole configuration that carries one unit of $T_{L}^{3}$ magnetic charge, where $T_{L}^{3}=\operatorname{diag}(1,-1)$ denotes the third generator of $S U(2)_{L}$. In other words, the monopole emits both electromagnetic (EM) and $Z$ magnetic flux, where

Published by the American Physical Society under the terms of the Creative Commons Attribution 4.0 International license. Further distribution of this work must maintain attribution to the author(s) and the published article's title, journal citation, and DOI. Funded by SCOAP . the latter appears in a flux tube (or $Z$ string). The EM and $Z$ magnetic flux carried by the monopole are found to be $(4 \pi / e) \sin ^{2} \theta_{w}$ and $(4 \pi / e) \sin \theta_{w} \cos \theta_{w}$, respectively, where $\theta_{w}$ is the electroweak mixing angle with $\sin ^{2} \theta_{w}=0.23$. This monopole cannot be purely electromagnetic in nature with the $Z$ flux screened within its core (size $\sim M_{Z}^{-1}$ ) because it would violate Gauss's law pertaining to the magnetic component of $U(1)_{Y}$.

A recent paper [6] shows that the Nambu monopole configurations routinely arise in GUTs, with the simplest example provided by $S U(5)$. A new feature here is the presence of an elementary scalar $S U(2)_{L}$ triplet with $Y=0$ that acquires an induced vacuum expectation value (VEV) from the electroweak breaking. In addition, the quantized value of the $Z$ flux is also predicted by exploiting the wellknown prediction of $\sin ^{2} \theta_{w}=3 / 8$.

Motivated by these considerations, it seems appropriate to enquire whether a purely electromagnetic monopole configuration can be realized in the SM. Clearly, this cannot happen without involving the $U(1)_{Y}$ sector, in contrast to the claim in Refs. $[7,8]$. Ignoring the singular nature of a Dirac monopole [9] for a moment, it turns out that a purely electromagnetic monopole can be realized by combining via $Z$ strings a single $Y$ monopole of appropriate magnetic charge with three Nambu monopoles. This composite monopole structure carries a magnetic charge of $(12 \pi / e)$ in order to satisfy the Dirac quantization conditions in the presence of quarks and charged leptons. All dependence on $\sin ^{2} \theta_{w}$ disappears in arriving at this 
conclusion. We show how the embedding of $U(1)_{Y}$ in a grand unified framework such as $S U(5)$ resolves the singular nature of the above composite monopole. As expected, the monopole turns out to be superheavy with mass comparable to the GUT scale. Two additional examples are provided of configurations that carry EM charges of $4 \pi / e$ and $8 \pi / e$ as well as screened color magnetic charge.

\section{COMBINING NAMBU AND DIRAC MONOPOLES IN THE STANDARD MODEL}

We are admitting the possibility of a $U(1)_{Y}$ Dirac monopole in the SM. From the Dirac quantization condition such a monopole carries magnetic hypercharge

$$
m_{Y}=\frac{12 \pi}{g^{\prime}} n, \quad n \in \mathcal{Z}
$$

where $g^{\prime}$ is the hypercharge gauge coupling constant, and the left-handed quark doublet $(u, d)_{L}$ has hypercharge $1 / 6$. Next consider a Nambu monopole in the symmetrybreaking $S U(2)_{L} \rightarrow U(1)_{L}$. Such a monopole has weak magnetic charge given by the condition

$$
m_{L}=\frac{2 \pi}{g / 2} n^{\prime}=\frac{4 \pi}{g} n^{\prime}, \quad n^{\prime} \in \mathcal{Z},
$$

where $g$ is the $S U(2)_{L}$ coupling constant. We can work in the unitary gauge for the $S U(2)_{L}$ monopole such that the magnetic flux from the monopole consists only of $W_{\mu}^{3}$ gauge field, where $W_{\mu}^{a}$ denote the usual $S U(2)_{L}$ gauge fields.

We now wish to put the Nambu $S U(2)_{L}$ and Dirac $U(1)_{Y}$ monopoles together and reexpress the charges in terms of the postelectroweak symmetry-breaking gauge fields: $Z_{\mu}$ and $A_{\mu}$. The relation between $\left(W_{\mu}^{3}, Y_{\mu}\right)$ and $\left(Z_{\mu}, A_{\mu}\right)$ is given by

$$
\begin{gathered}
W_{\mu}^{3}=\cos \theta_{w} Z_{\mu}+\sin \theta_{w} A_{\mu}, \\
Y_{\mu}=\cos \theta_{w} A_{\mu}-\sin \theta_{w} Z_{\mu} .
\end{gathered}
$$

Therefore, the $Z_{\mu}$ and $A_{\mu}$ magnetic charges on $n^{\prime} S U(2)_{L}$ monopoles are

$$
m_{L Z}=\frac{4 \pi n^{\prime}}{g} \cos \theta_{w}, \quad m_{L A}=\frac{4 \pi n^{\prime}}{g} \sin \theta_{w},
$$

and on $n U(1)_{Y}$ monopoles are

$$
m_{Y Z}=-\frac{12 \pi n}{g^{\prime}} \sin \theta_{w}, \quad m_{Y A}=\frac{12 \pi n}{g^{\prime}} \cos \theta_{w} .
$$

The net $Z_{\mu}$ and $A_{\mu}$ magnetic charges on a conglomerate of $n U(1)_{Y}$ and $n^{\prime} S U(2)_{L}$ monopoles are

$$
\begin{aligned}
& m_{Y+L, Z}=\frac{4 \pi n^{\prime}}{g} \cos \theta_{w}-\frac{12 \pi n}{g^{\prime}} \sin \theta_{w} \\
& m_{Y+L, A}=\frac{4 \pi n^{\prime}}{g} \sin \theta_{w}+\frac{12 \pi n}{g^{\prime}} \cos \theta_{w} .
\end{aligned}
$$

This configuration should not have any net $Z$ magnetic charge because the $Z$ magnetic fields are confined once the electroweak symmetry is broken. Any net $Z$ flux would form a string that would confine the monopole conglomerate to an anticonglomerate. Thus, we require

$$
\frac{4 \pi n^{\prime}}{g} \cos \theta_{w}-\frac{12 \pi n}{g^{\prime}} \sin \theta_{w}=0 .
$$

Together with the relations

$$
\cos \theta_{w}=\frac{g}{\sqrt{g^{2}+g^{\prime 2}}}, \quad \sin \theta_{w}=\frac{g^{\prime}}{\sqrt{g^{2}+g^{\prime 2}}},
$$

the constraint in (9) gives

$$
n^{\prime}=3 n,
$$

and so the conglomerate should contain three times as many Nambu monopoles as the Dirac $Y$ monopoles.

Next let us consider the EM magnetic charge on the conglomerate. From (8), taking into account (10) and (11), we get

$$
m_{Y+L, A}=\frac{12 \pi}{e} n,
$$

where $e=g g^{\prime} / \sqrt{g^{2}+g^{\prime 2}}$ is the electromagnetic coupling constant. Therefore the minimal EM charge of the conglomerate is $6 \times 2 \pi / e$ and this result is independent of $\sin ^{2} \theta_{w}$.

In Fig. 1 we sketch the minimal allowed monopole conglomerate. From (5), we see that the minimal $\left(n^{\prime}=1\right)$ magnetic flux along the $Z$ string is $4 \pi / g_{z}$, where $g_{z}=g / c_{w}$ is the gauge coupling associated with the $Z$ gauge boson with $c_{w}=\cos \theta_{w}$. This string corresponds to a $4 \pi$ rotation about the corresponding generator $T_{L}^{3} / 2-s_{w}^{2} Q$ and therefore it carries magnetic flux $T_{L}^{3}-2 s_{w}^{2} Q$, where $s_{w}=\sin \theta_{w}$. The minimal Nambu monopole also carries EM magnetic charge $(4 \pi / g) s_{w}=(4 \pi / e) s_{w}^{2}$ corresponding to magnetic flux $2 Q s_{w}^{2}$.

In Sec. IV we show how a monopole configuration with EM charge $4 \pi / e$ is realized by merging a single Nambu monopole with a colored $U(1)_{Y}$ monopole.

\section{NAMBU AND $U(1)_{Y}$ MONOPOLES IN $S U(5)$}

The embedding of the SM in a grand unified framework has important ramifications, especially as far as our current discussion is concerned. For instance, in minimal $S U(5)$ 


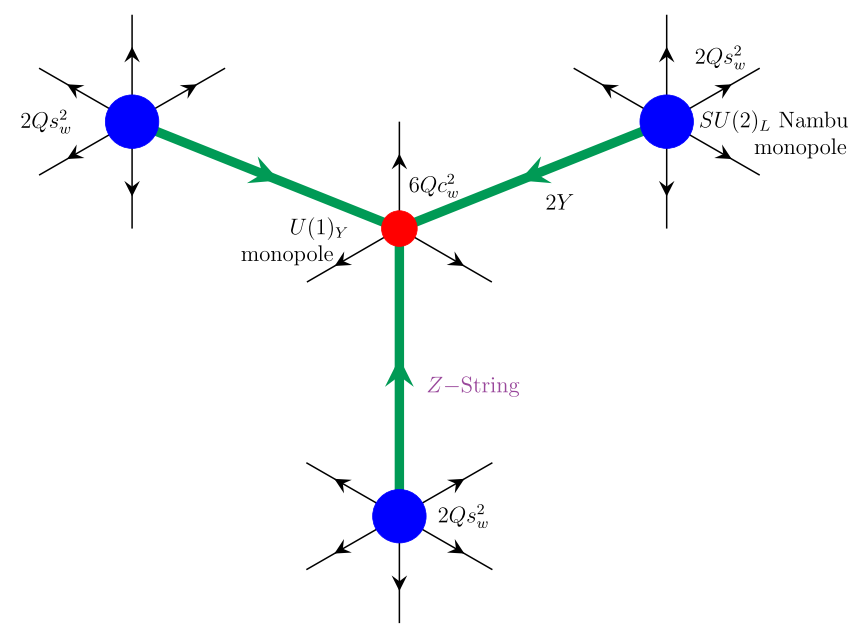

FIG. 1. A $U(1)_{Y}$ Dirac monopole (red) with minimal EM magnetic flux $6 Q c_{w}^{2}$, where $c_{w}=\cos \theta_{w}$, gets attached to three Nambu $S U(2)_{L}$ monopoles (blue) each with EM magnetic flux $2 Q s_{w}^{2}$, where $s_{w}=\sin \theta_{w}$. The $Z$ component of the $S U(2)_{L}$ flux of the Nambu monopoles is confined in strings with flux $T_{L}^{3}-2 s_{w}^{2} Q$ that connect them to the $U(1)_{Y}$ Dirac monopole. The conglomerate yields an electromagnetic monopole that carries six quanta $(12 \pi / e)$ of EM magnetic charge.

the Nambu monopole contains an inner core due to the presence of a heavy $S U(2)_{L}$ scalar triplet field that acquires an induced VEV through mixing with the SM Higgs doublet. The Nambu monopole carries $(3 / 4)(2 \pi / e)$ unit of Dirac magnetic charge, and the $Z$ magnetic flux is also predicted [6]. The composite electromagnetic monopole discussed above is now realized by considering a $U(1)_{Y}$ monopole with winding number six, which appears from the symmetry breaking $S U(5) \rightarrow S U(3)_{c} \times S U(2)_{L} \times$ $U(1)_{Y} / Z_{3} \times Z_{2}$. At this stage the monopole has a core size of order $M_{\mathrm{GUT}}^{-1}$ and mass around $10 M_{\mathrm{GUT}}$. Clearly, the singularity encountered with such a monopole in the SM setting has now disappeared.

Following electroweak breaking the monopole carries an appropriate combination of EM and $Z$ magnetic fluxes, with the latter in the form of $Z$ strings. In order to retain a finite energy configuration this $U(1)_{Y}$ monopole in $S U(5)$ must merge with three Nambu monopoles to yield a composite structure that carries six quanta of Dirac magnetic charge (Fig. 1). Note that in the absence of quarks just two quanta $(4 \pi / e)$ would have been compatible with the Dirac quantization condition. Indeed, in the $S U(5)$ breaking to $S U(3)_{c} \times S U(2)_{L} \times U(1)_{Y} / Z_{3} \times Z_{2}$ the fundamental topologically stable monopole carries $S U(3)_{c}, S U(2)_{L}$, and $U(1)_{Y}$ magnetic charges. The subsequent breaking of the SM symmetry to $S U(3)_{c} \times U(1)_{\mathrm{em}} / Z_{3}$ yields a monopole carrying a single quantum $(2 \pi / e)$ of Dirac magnetic charge as well as screened color magnetic charge. Quark confinement in this case is required in order for the Dirac quantization condition to be satisfied beyond the screening radius $\sim \Lambda_{\mathrm{QCD}}^{-1}$.
Finally, let us note that a purely electromagnetic monopole carrying three quanta of Dirac magnetic charge and accompanied by no other field exists in $S U(5)$, and a classical solution has been shown in Ref. [10].

\section{COLORED DIRAC MONOPOLES}

The smallest homotopically nontrivial loop in the SM gauge group $S U(3)_{c} \times S U(2)_{L} \times U(1)_{Y} / Z_{3} \times Z_{2}$ consists of a $2 \pi$ rotation about $Y$-for the left-handed quark doublet $Y=1 / 6$ - accompanied by a $\pi$ rotation about $T_{L}^{3}=$ $\operatorname{diag}(1,-1)$ and a $2 \pi / 3$ rotation about $T_{c}^{8}=\operatorname{diag}(1,1,-2)$. The minimal singular Dirac monopole then has magnetic flux $Y+T_{L}^{3} / 2+T_{c}^{8} / 3$. The magnetic flux of the doubly charged Dirac monopole can be written as $2 Y+2 T_{c}^{8} / 3$ since the loop in $S U(2)_{L}$ becomes homotopically trivial and can be removed. After electroweak symmetry breaking, the colored Dirac monopole can absorb the $Z$ flux emerging from the Nambu monopole (Fig. 2) (see also Ref. [11]). The total EM plus color flux of the conglomerate is $2 Q+2 T_{c}^{8} / 3$ with the color part screened for distances larger than the QCD scale.

\section{STRUCTURE AND STABILITY}

Figures 1 and 2 show the purely EM monopoles in terms of their constituent Dirac and Nambu monopoles. However, we do not expect these diagrams to realistically show classical solutions of the field theory. For example, the colored monopole of Fig. 2 is more likely to be a structureless, pointlike $S U(3) \times U(1)_{Y} / Z_{3}$ Dirac monopole surrounded by a cloud of electroweak fields that make up the Nambu monopole. Other solutions have been proposed in Ref. [12] though these solutions do not correspond to the correctly quantized monopoles depicted in Figs. 1 and 2-the conglomerate in Fig. 1 has three (not one) Nambu monopoles, and the conglomerate in Fig. 2 has net color charge.

If the Dirac monopoles are regularized as $S U(5)$ monopoles, Fig. 2 corresponds to the winding 2 monopole

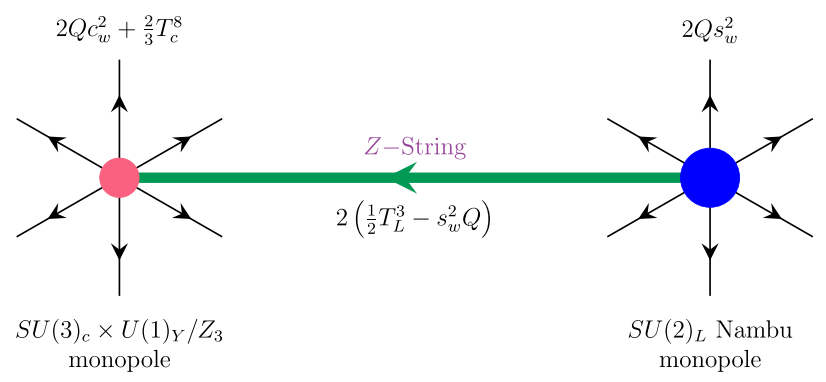

FIG. 2. A doubly charged colored Dirac monopole (pink) and a Nambu monopole (blue) connected by a $Z$ string with magnetic flux $T_{L}^{3}-2 s_{w}^{2} Q$. The total flux of this configuration is $2 Q+2 T_{c}^{8} / 3$ of which the colored part will be screened due to QCD effects. 
resulting from the symmetry breaking to $S U(3)_{c} \times$ $S U(2)_{L} \times U(1)_{Y} / Z_{3} \times Z_{2}$, while Fig. 1 corresponds to the winding 6 grand unified monopole. The odd winding $S U(5)$ monopoles carry $S U(2)_{L}$ magnetic charge and once electroweak symmetry breaks; two odd winding monopoles combine to result in an $S U(2)_{L}$ neutral monopole [13]. Further, it has been shown that the $S U(5)$ monopole with winding $n=5$ is unstable to fragmentation into $n=2+3$ monopoles, and monopoles with winding $n \geq 7$ are unstable to fragmentation into $n=6+(n-6)$ monopoles $[14,15]$. Then the only other relevant winding to consider in the $S U(5)$ regularization is $n=4$. This monopole will connect with two Nambu monopoles to form a conglomerate that carries color and EM magnetic charges but the color magnetic field will get screened to give a pure EM monopole with flux $4 Q$ (EM charge $8 \pi / e$ ) at large distances.

\section{SUMMARY}

We have explored how purely EM monopole configurations may arise in the SM. We do not expect such configurations to have well-defined energy because of the singular nature of the Dirac monopole associated with the $U(1)_{Y}$ sector of the electroweak model. Barring this, we identified a configuration consisting of a single $U(1)_{Y}$ Dirac monopole which is linked via $Z$ strings to three Nambu monopoles. This composite monopole carries six units $(12 \pi / e)$ of EM magnetic charge in order to be compatible with the Dirac quantization condition in the presence of quarks. Although the $Y$ and Nambu monopoles both carry $\theta_{w}$ -dependent EM and $Z$ magnetic charges, the end result leaves $\theta_{w}$ undetermined in the $S U(2)_{L} \times U(1)_{Y} / Z_{2}$ framework. We also show how this singular composite monopole gets regularized and obtains a finite, albeit superheavy mass, from embedding the SM in a GUT. In the second example a colored $U(1)_{Y}$ monopole merges with a single Nambu monopole to yield a composite topological structure carrying an EM magnetic charge of $4 \pi / e$ as well as screened color magnetic charge. A third example consists of two Nambu monopoles and a single colored Dirac $U(1)_{Y}$ monopole and has EM magnetic charge $8 \pi / e$.

Let us recall here that models such as $S U(4)_{c} \times$ $S U(2)_{L} \times S U(2)_{R}$ [16] and $S U(3)_{c} \times S U(3)_{L} \times S U(3)_{R}$ predict electric charge quantization [17,18], but gauge coupling unification in these models is not automatic.
In this case the masses of the composite monopoles can lie in the multi-TeV range. It is worth stressing that these two models also predict the existence of topologically stable magnetic monopoles that, respectively, carry two and three Dirac quanta of magnetic charge, and which may be accessible at high-energy colliders.

Before concluding we offer some remarks regarding the observability of these composite monopoles at the LHC and future high-energy colliders. The production cross section of a composite coherent quantum state including these composite monopole states is expected to be exponentially suppressed in Drell-Yan processes involving particle collisions [19]. This is reminiscent of the exponential suppression in quantum-mechanical tunneling. It has been argued that the magnetic analog of the Schwinger mechanism could be exploited to significantly enhance the production cross sections of these extended structures and propel them in the observable range. With adequately strong magnetic fields the (dual) Schwinger mechanism may lead to an observable cross section for monopole pair production in heavy ion collisions [20]. The MoEDAL experiment has recently presented the first results [21] based on this idea.

For the composite monopoles to be accessible at highenergy colliders their mass should lie in the $\mathrm{TeV}$ range or so. This means that the $U(1)_{Y}$ component of the electroweak sector of the SM should be embedded in a unifying gauge symmetry such as $S U(3)_{L} \times S U(3)_{R}$, which may be broken at the $\mathrm{TeV}$ scale if we do not require gauge coupling unification of the left-right $S U(3)$ symmetries [18,22]. For a discussion of monopoles in cosmic rays, see Ref. [22] and other references listed there.

\section{ACKNOWLEDGMENTS}

We thank Amit Tiwari for drawing the figures and P. Q. Hung and Nick Mavromatos for discussions. This work is supported by the Hellenic Foundation for Research and Innovation (H. F. R. I.) under the "First Call for H. F. R. I. Research Projects to support Faculty Members and Researchers and the procurement of high-cost research equipment grant" (Project No. 2251). Q. S. is supported in part by the DOE Grant No. DE-SC-001380. T. V. is supported by the U.S. Department of Energy, Office of High Energy Physics, under Award No. DE-SC0019470 at ASU.
[1] Y. Nambu, String-like configurations in the WeinbergSalam theory, Nucl. Phys. B130, 505 (1977).

[2] T. Vachaspati, Vortex Solutions in the Weinberg-Salam Model, Phys. Rev. Lett. 68, 1977 (1992); Erratum, Phys. Rev. Lett. 69, 216 (1992).
[3] A. Achucarro and T. Vachaspati, Semilocal and electroweak strings, Phys. Rep. 327, 347 (2000).

[4] G. 't Hooft, Magnetic monopoles in unified gauge theories, Nucl. Phys. B79, 276 (1974). 
[5] A. M. Polyakov, Particle spectrum in the quantum field theory, JETP Lett. 20, 194 (1974).

[6] G. Lazarides and Q. Shafi, Electroweak monopoles and magnetic dumbbells in grand unified theories, Phys. Rev. D 103, 095021 (2021).

[7] P. Q. Hung, Topologically stable, finite-energy electroweakscale monopoles, Nucl. Phys. B962, 115278 (2021).

[8] J. Ellis, P. Q. Hung, and N. Mavromatos, An electroweak monopole, Dirac quantization and the weak mixing angle, Nucl. Phys. B969, 115468 (2021).

[9] P. A. M. Dirac, Quantised singularities in the electromagnetic field, Proc. R. Soc. A 133, 60 (1931).

[10] V. A. Rubakov and M. S. Serebryakov, Anomalous baryon number non-conservation in the presence of $S U(5)$ monopoles, Nucl. Phys. B218, 240 (1983).

[11] J. Preskill, Semilocal defects, Phys. Rev. D 46, 4218 (1992).

[12] Y. M. Cho and D. Maison, Monopoles in Weinberg-Salam model, Phys. Lett. B 391, 360 (1997).

[13] Y. Ng, T. W. B. Kibble, and T. Vachaspati, Formation of non-Abelian monopoles connected by strings, Phys. Rev. D 78, 046001 (2008).

[14] T. Vachaspati, An Attempt to Construct the Standard Model with Monopoles, Phys. Rev. Lett. 76, 188 (1996).
[15] H. Liu and T. Vachaspati, $S U(5)$ monopoles and the dual standard model, Phys. Rev. D 56, 1300 (1997).

[16] J. C. Pati and A. Salam, Lepton number as the fourth color, Phys. Rev. D 10, 275 (1974); Erratum, Phys. Rev. D 11, 703 (1975).

[17] G. Lazarides and Q. Shafi, Monopoles, strings, and necklaces in $S O(10)$ and $E_{6}$, J. High Energy Phys. 10 (2019) 193.

[18] G. Lazarides and Q. Shafi, Triply charged monopole and magnetic quarks, Phys. Lett. B 818, 136363 (2021).

[19] N. E. Mavromatos and V. A. Mitsou, Magnetic monopoles revisited: Models and searches at colliders and in the Cosmos, Int. J. Mod. Phys. A 35, 2030012 (2020).

[20] O. Gould, D. L. J. Ho, and A. Rajantie, Schwinger pair production of magnetic monopoles: Momentum distribution for heavy-ion collisions, Phys. Rev. D 104, 015033 (2021).

[21] B. Acharya, J. Alexandre, P. Benes, B. Bergmann, S. Bertolucci et al., First experimental search for production of magnetic monopoles via the Schwinger mechanism, arXiv:2106.11933.

[22] T. W. Kephart, G. K. Leontaris, and Q. Shafi, Magnetic monopoles and free fractionally charged states at accelerators and in cosmic rays, J. High Energy Phys. 10 (2017) 176. 